\title{
University of Southampton Library Holding ISBN: 9788366675599
}

Hartley Library / University of Southampton

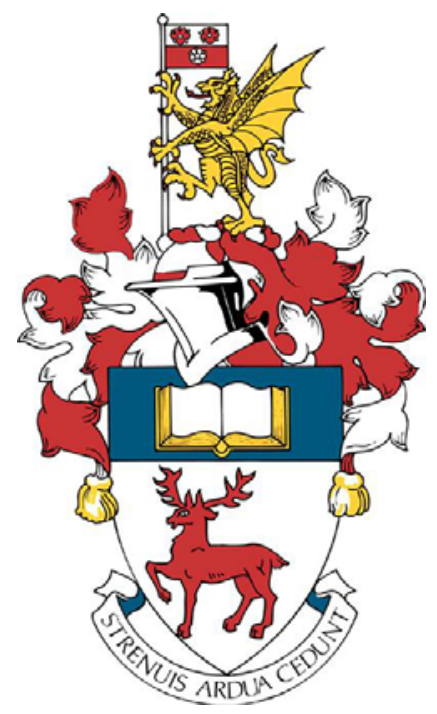

Hartley Library B12, University Rd, Highfield, Southampton S017 1BJ, United Kingdom Library Holding:

\section{Southampintorn}

WebCat: library catalogue of the University of Southampton

Search/Home Other Catalogues Course Collections My Account

\section{Go Back Help New Search Print/email marked records Permalink Logout}

record 1 of 1 for search keywords "mindsponge-based"

\section{Item Details}

$\square$ Mark

Find more by this author

Nearby items on shelf \begin{tabular}{|l|l|l|l|l|l|}
\hline Copy details & Contents/Summary & Full Calogue Details
\end{tabular}

A Mindsponge-Based Investigation into the Psycho-Religious Mechanism Behind Suicide Attacks Vuong, Quan-Hoang, author

ISBN:

9788366675599

Other ID:

$10.2478 / 9788366675599$ doi

Personal Author:

Vuong, Quan-Hoang, author.

A Mindsponge-Based Investigation into the

Psycho-Religious Mechanism Behind Suicide

Attacks / Quan-Hoang Vuong, Minh-Hoang

Nguyen, Tam-Tri Le.

Copyright notice: Warsaw ; Berlin : Sciendo, [2021]

Copyright notice: $\quad$ (C)2021

Physical description: 1 online resource (127 p.)

Contents note: $\quad$ Frontmatter -- Preface -- Acknowledgment --

Table of Contents -- About the Authors --

Abbreviations and acronyms -- List of Figures

-- List of Tables -- Chapter 1: Suicide

attacks: trends and psychological research --

Chapter 2: Religions and the end of life - 


\section{Navigation Menu \\ Search/Home \\ Other Catalogues \\ Course Collections \\ My Account}

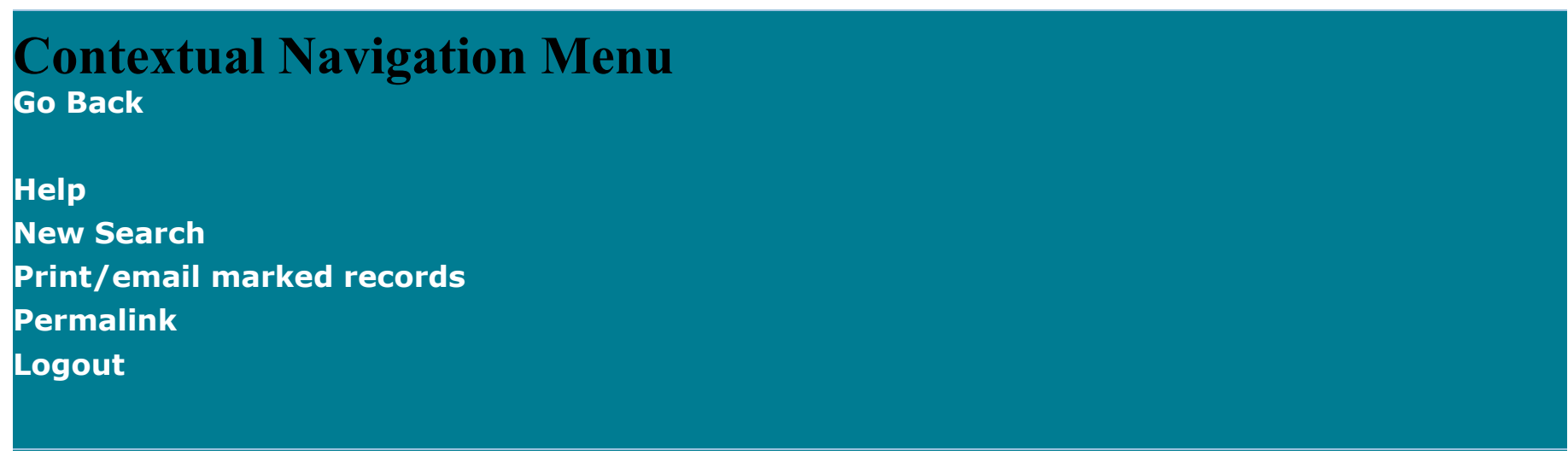

Item Details

\begin{tabular}{l|l|} 
Copy details & Contents/Summary \\
\hline
\end{tabular}

Full Catalogue Details MARC Record

A Mindsponge-Based Investigation into the Psycho-Religious Mechanism Behind Suicide Attacks

Vuong, Quan-Hoang, author.

ISBN:

9788366675599

Other ID:

10.2478/9788366675599 doi

Personal Author:

Vuong, Quan-Hoang, author.

Title:

A Mindsponge-Based Investigation into the Psycho-Religious Mechanism Behind Suicide Attacks / QuanHoang Vuong, Minh-Hoang Nguyen, Tam-Tri Le.

Copyright notice:

Warsaw ; Berlin : Sciendo, [2021]

Copyright notice:

(C)2021

Physical description:

1 online resource (127 p.)

Contents note:

Frontmatter -- Preface -- Acknowledgment -- Table of Contents -- About the Authors -- Abbreviations and acronyms -- List of Figures -- List of Tables -- Chapter 1: Suicide attacks: trends and psychological research -- Chapter 2: Religions and the end of life -- Chapter 3: Mindsponge mechanism -- Chapter 4: Investigation into the psycho-religious mechanism -- Chapter 5: Psycho-religious mechanism -- Chapter 6: The (ir)rationality behind extreme behaviors -- Chapter 7: Terrorism prevention through trust-building -- Closing remarks -- Appendix A -- Appendix B -- References -- Index

Access restriction:

restricted access http://purl.org/coar/access_right/c_16ec online access with authorization star Local note:

E-book: full text accessible via the link below (authenticated).

Language note:

In English.

Source Description:

Description based on online resource; title from PDF title page (publisher's Web site, viewed 29. Jul 2021)

Added author:

Le, Tam-Tri, author.

Added author:

Nguyen, Minh-Hoang, author.

Electronic access:

Click for access 
Online Form:

Cover : Click for access 
*Permalink:

https://www-lib.soton.ac.uk/uhtbin/cgisirsi/?ps=6ied4AoBdw/HARTLEY/X/123

(Accessed: September 19, 2021) 